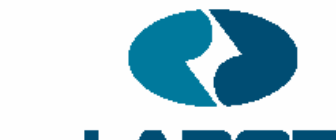

LABORATORIO RICCARDO REVELLI

GENTRE FOA EMPLOYMINT ETUDIE.

Working Paper no. 109

\title{
Sequential Teamwork in Competitive Environments: Theory and Evidence from Swimming Data
}

\author{
Michael Neugart \\ Free University of Bozen/Bolzano, School of Economics and Management \\ Matteo G. Richiardi \\ University of Turin, and LABORatorio R. Revelli, Turin
}

May, 2011

Laboratorio R. Revelli, Collegio Carlo Alberto

Via Real Collegio, 30 - 10024 Moncalieri (TO)
Tel. +39011670.50.60 - Fax +39011670.50.61 www.laboratoriorevelli.it - labor@laboratoriorevelli.it 


\title{
Sequential Teamwork in Competitive Environments: Theory and Evidence from Swimming Data
}

\author{
Michael Neugart* and Matteo G. Richiardi ${ }^{\dagger}$
}

May 2011

\begin{abstract}
The aim of the paper is to assess whether there is free-riding in teams when team production is sequential and when there is competition between teams. This a common case, which, however, has not been considered in the literature so far. We develop a model where team members contributing earlier have an incentive to free-ride more even when there is competition between teams. These predictions are tested on more than 300.000 observations on swimmers' performance at competitions from all over the world. We find that swimmers in relays perform weaker as compared to their individual performance, and that earlier swimmers' performance in relays is weaker relative to later swimmers. Our results suggest that competition does not solve the free-riding problem in team production with sequential contributions.
\end{abstract}

Keywords: team production, contest, intergroup competition, sequential contribution, free-riding, swimming

JEL-Classification: C70, D20, D70, H40

\section{Introduction}

In this contribution we look into team production when team members sequentially contribute to the team, and teams compete against each other for a prize.

Team production is a fundamental characteristic of modern societies. Many activities require the joint effort of a multitude of participants and so teamwork has become prevalent in many firms. ${ }^{1}$ The specific organization of teams in firms depends on the nature of tasks that have to be accomplished. Some tasks may

\footnotetext{
${ }^{*}$ Free University of Bozen/Bolzano, School of Economics and Management, Piazza Università 1, I-39100 Bozen, Italy, e-mail: Michael.Neugart@unibz.it

${ }^{\dagger}$ University of Turin, Department of Economics, and LABORatorio Revelli, via Po 53, I-10124 Torino, Italy, e-mail: matteo.richiardi@unito.it

${ }^{1}$ From 1987 to 1996 the share of firms having more than $20 \%$ of employees organized in teams rose from $37 \%$ to $66 \%$ (Lawler et al., 2001; Lazear and Shaw, 2007).
} 
require the simultaneous contribution of team members, other very common situations entail subsequent production phases that require sequential contributions by team members, where each contribution builds on previous work done by the other team members. Irrespective of the scope for which teams are used, it is well know that it can be time consuming to coordinate and organize them. Moreover, team production might be plagued by disincentive problems because individuals free-ride on the contributions of other team members (Alchian and Demsetz, 1972; Holmstrom, 1982), something the managers of teams and team members themselves constantly worry about.

How to solve the free-riding problem becomes key, and letting teams compete may alleviate it. In a laboratory experiment Bornstein et al. (1990) compared the performance of groups in a social dilemma situation under two conditions: one in which groups were not facing competition and another in which groups were competing for an additional reward. They found that between-group competition significantly increased the contributions of the simultenously acting team members, a finding that was replicated by Erev et al. (1993) in a different work environment where subjects picked oranges, as well as by Gunnthorsdottir and Rapoport (2006).

In an experimental study Erev and Rapoport (1990) compared sequential and simultaneous contributions to public goods and showed that simultaneous choice is significantly less effective in solving the dilemma. Contrarily, Varian (1994) argued that with sequential choice the free-riding problem is exacerbated with respect to the simultaneous contributions mechanism, and that there exists a first mover advantage with early contributors contributing less. Experimental evidence specifically targeting Varian (1994) by Andreoni et al. (2002) confirms the first mover behavior but also states that the difference between simultaneous and sequential play vanishes to the end of the experiment, while more recent experiments by Gaechter et al. (2009) support the prediction that the overall contribution is lower in sequential contributions but do not find evidence for the predicted first mover advantage. None of this work, however, considers the effect of competition between groups on within-group performance. In summary, surprisingly, little seems to be known on whether competition between teams eliminates free-riding as team members contribute sequentially.

The present study tries to shrink this gap in the literature with two contributions. We first develop a model where members of competing teams contribute sequentially to win a commonly shared price. Secondly, we provide an empirical assessment of free-riding within teams when there is competition between teams.

Developing our model we draw on the literature on contests between groups, following Katz et al. (1990), Ursprung (1990), or Gradstein (1993) among others, who model competition between groups when group members contribute simultaneously. ${ }^{2}$ Starting from these studies we delineate a model of competition between groups when team members contribute sequentially to the team. There is a prize for the winning team awarded to every team member. Higher costly efforts by

\footnotetext{
${ }^{2}$ Two recent surveys are Corchón (2007) and Konrad (2009).
} 
any team member increases the probability for the team to win the price, as team members efforts' enter additively a Tullock contest function.

For testing our predictions we turn to swimming data. ${ }^{3}$ Typically, it is difficult to measure the performance of workers and her individual contribution to a team in standard work situations. Moreover, from a researcher's perspective it is usually infeasible to construct a convincing counterfactual that would allow to draw causal inference from the observations in an environment of an operating firm. In order to overcome these problems economists have increasingly turned to sports data recognizing that these markets provide a number of natural experiments which allow for the testing of the effects of incentives on labour-market behavior (Kahn, 2000; Szymanski, 2003). Along these lines, a well known attempt linking contest theory with data from sports is Ehrenberg and Bognanno (1990) who examined the size of prize funds on the scores of golfers at major tournaments.

Our comprehensive dataset covers swimming competitions from all over the world during the years 1972 to 2009 with a total of more than 300,000 observations. It seems to be particularly suited for our purposes as it allows us to construct a counterfactual for each individual's performance by comparing times swam in individual races with the same swimmer's performance in a relay at the same event typically taking place within a time period smaller than 2 days. The evidence supports the prediction that competition between teams does not wash out the freeriding problem that typically arises with team production. Moreover, the data also confirms that team members moving earlier free-ride more.

We proceed in the following way. In section 2 we set-up the theoretical model. In section 3 we present our empirical testing strategy, providing some background on the rules and main characteristics of swimming competitions and describing the data we use, while in section 4 we present and discuss our empirical results. In section 5 we conclude.

\section{A model of inter-group competition with sequential intra- group choices}

We model competition between groups along the lines of contest theory which goes back to the seminal contribution by Tullock (1980). Tullock employed a contest success function (CSF) where the probability of winning the competition is equal to the ratio of own effort to global effort, the sum of efforts of all contenders. ${ }^{4}$

There are two teams $A$ and $B$ competing for a prize $S$. Each team has two

\footnotetext{
${ }^{3}$ Existing studies employing swimming data mostly involve experimental work stemming from the area of social psychology with one of the earliest contributions by Sorrentino and Sheppard (1978), followed by Williams et al. (1989), Everett et al. (1992) or Miles and Greenberg (1993).

${ }^{4}$ Tullock's idea was to compare rent seeking activity -group contribution in our setting- to the purchase of lottery tickets: the higher the number of tickets, the more likely to win the lottery. Skaperdas (1996) provided an axiomatic foundation for the Tullock CSF, while more recently Jia (2008) offered a distribution based justification for its ratio form.
} 
players, denoted with 1 and 2. All players are of homogeneous ability. The prize $S$ has equal value to each of the team members.

Team members exert effort $e$ at cost $c(e)$. We employ a quadratic cost function $c(e)=e^{2}$. As in Gunnthorsdottir and Rapoport (2006) the contributions of team members to the overall team output enter additively. To reflect the sequential nature of the game, however, first members of teams $A$ and $B$ choose their effort level first. Second players make their choice on the basis of first players' outcome. Thus, members 1 are Stackelberg leaders vis à vis members 2 in their teams. We denote with $e_{A 1}$ effort spent by the team member moving first in team $A$, and $e_{A 2}$ as effort spent by the team member who moves second in team $A$. Notation for team $B$ is accordingly.

The fact that effort is not deterministically transformed into performance, and that there are stochastic elements in the competition is taken into consideration by CSFs. In our setting, however, uncertainty is partly resolved after the first players finish their task. Hence, second players face a different informational content. We model this by introducing an additional random term $\varepsilon$ with support $[0,1]$. Realization of this random variable takes place before second players choose their effort, and is thus considered as given for second players, as is first players' effort. This amounts to say that second players observe first players' performance.

The winning probability of team $A$ is then

$$
p_{A}= \begin{cases}\alpha \frac{e_{A 1}+e_{A 2}}{e_{A 1}+e_{A 2}+e_{B 1}+e_{B 2}}+(1-\alpha) \varepsilon & \text { if } e_{A 1}+e_{A 2}+e_{B 1}+e_{B 2}>0 \\ \frac{1}{2} & \text { otherwise, }\end{cases}
$$

with $0<\alpha<1$ being a relative weighting factor of the random component on individual performance. The probability of winning for the other team $B$ is $p_{B}=$ $1-p_{A}$.

Expected payoffs for the second players are

$$
\begin{aligned}
& V_{A 2}=p_{A} S-e_{A 2}^{2} \\
& V_{B 2}=p_{B} S-e_{B 2}^{2}
\end{aligned}
$$

where the realization of $\varepsilon$ is known to them. Accordingly expected payoffs for first players who do not know the realization of $\varepsilon$ at the time they make their choice are

$$
\begin{aligned}
& V_{A 1}=p_{A} S-e_{A 1}^{2} \\
& V_{B 1}=p_{B} S-e_{B 1}^{2} .
\end{aligned}
$$

The game is solved by backward induction. Second players take the effort level of first players as given, know the realization of $\varepsilon$, and choose their own effort simultaneously. First players make their choices taking into account the later realization of $\varepsilon$, and the reaction of the second players in teams $A$ and $B$, respectively.

Everything being homogenous from the point of view of first players, symmetry is expected, i.e. $e_{A 1}^{*}=e_{B 1}^{*}$ and $e_{A 2}^{*}=e_{B 2}^{*}$. Symmetry is potentially broken for 
the second players, as the realization of $\varepsilon$ is known. However, given that $\varepsilon$ enters additively the CSF, its value does not affect the choices of second players, and symmetry is restored. Hence, everybody expects that simultaneous players will put the same effort. Imposing $e_{A 1}^{*}=e_{B 1}^{*}$ and $e_{A 2}^{*}=e_{B 2}^{*}$, as we will do in the following, greatly simplifies the derivation of the proofs. ${ }^{5}$

For second players, effort choices follow from the first order conditions

$$
\begin{aligned}
& H \equiv \frac{\mathrm{d} V_{A 2}}{\mathrm{~d} e_{A 2}}=\alpha \frac{e_{B 1}+e_{B 2}}{D^{2}} S-2 e_{A 2}=0 \\
& F \equiv \frac{\mathrm{d} V_{B 2}}{\mathrm{~d} e_{B 2}}=\alpha \frac{e_{A 1}+e_{A 2}}{D^{2}} S-2 e_{B 2}=0
\end{aligned}
$$

with $D=e_{A 1}+e_{A 2}+e_{B 1}+e_{B 2}$. Expected marginal gains, equal to the sum of the marginal increase in the probability of winning as effort is increased times the valuation of the prize $S$, have to equal the marginal costs. Solving these first order conditions for the optimal choices $e_{A 2}^{*}$ and $e_{B 2}^{*}$, ruling out the negative effort choices, yields

$$
\begin{aligned}
& e_{A 2}^{*}=-\frac{e_{A 1}}{2}+\sqrt{\frac{e_{A 1}^{2}}{4}+\alpha \frac{S}{8}} \\
& e_{B 2}^{*}=-\frac{e_{B 1}}{2}+\sqrt{\frac{e_{B 1}^{2}}{4}+\alpha \frac{S}{8}} .
\end{aligned}
$$

With second order conditions being fulfilled, $e_{A 2}^{*}$ and $e_{B 2}^{*}$ constitute the best choice of the team members moving second.

Equations (8) and (9) are the reactions function which the first players in the team are facing with respect to the effort choices of their teammates who follow. Both reaction functions are downward sloping. An increase of effort of the first player leads to a decrease in the teammate's effort. Thus, effort choices within teams are substitutes, and it is this property which will essentially drive our result that second players exert more effort as we compare optimal choices within teams for first and second players.

First players of teams $A$ and $B$ also decide on their effort simultaneously taking into account that their particular decision will have an impact on all subsequent swimmers, and not knowing the realization of $\varepsilon$. The first order conditions for these players after taking expectations over $\varepsilon$ are

$$
\begin{aligned}
& \frac{\mathrm{d} V_{A 1}}{\mathrm{~d} e_{A 1}}=\alpha \frac{\left(e_{B 1}+e_{B 2}\right)\left(1+\frac{\mathrm{d} e_{A 2}}{\mathrm{~d} e_{A 1}}-\frac{\mathrm{d} e_{B 2}}{\mathrm{~d} e_{A 1}}\right)}{D^{2}} S-2 e_{A 1}=0 \\
& \frac{\mathrm{d} V_{B 1}}{\mathrm{~d} e_{B 1}}=\alpha \frac{\left(e_{A 1}+e_{A 2}\right)\left(1+\frac{\mathrm{d} e_{B 2}}{\mathrm{~d} e_{B 1}}-\frac{\mathrm{d} e_{A 2}}{\mathrm{~d} e_{B 1}}\right)}{D^{2}} S-2 e_{B 1}=0
\end{aligned}
$$

\footnotetext{
${ }^{5}$ The same results can be obtained without this assumption. The proofs are available upon request.
} 
using $e_{A 1}^{*}+e_{A 2}^{*}=e_{B 1}^{*}+e_{B 2}^{*}$. Eqs. (10) and (11) determine the effort choices of first players. Again, expected marginal benefits have to equal the marginal costs which are a function of the effort of the first players. Compared to the first order conditions of the second players, the marginal benefits of the first players take account of the effect of the effort choice of the first player on the teammates choice coming after, and the second player's effort choice of the competing team.

Proposition 1 In equilibrium, the optimal effort level provided by first players is lower than the effort level provided by second players.

Proof See Appendix A.

This result stems from the substitutability of within team members efforts, $-1<\frac{\mathrm{d} e_{A 2}}{\mathrm{~d} e_{A 1}}<0$, and $\frac{\mathrm{d} e_{B 2}}{\mathrm{~d} e_{A 1}}=0$ which implies that the choices of the first players do not have an impact on the other team's second player behavior. While the latter result is essentially driven by the symmetry of the set-up, the former is due to the substitutability of efforts within teams, as seen by the downward sloping reaction functions (8) and (9) The team member moving first knows that an increase in his effort is leading to a decrease in the effort of the team member moving second. Thus higher effort on his side is not fully reflected in a larger chance of winning the competition but he would still have to burden the higher costs of effort.

So far we have highlighted that first players free-ride with respect to second players, i.e. they exert a lower effort. Now, we show that also the effort of second players is too low with respect to the one that would be optimal for the team as a whole.

Proposition 2 In equilibrium, the effort level provided by second players is lower than the cooperative social optimum for the team.

Proof See Appendix A.

Intuitively, the cooperative solution takes into consideration the fact that the prize $S$ is non-rival and that effort exerted by any individual player has a positive externality on the team member. As marginal costs are increasing, it is advisable from a social point of view to share the burden equally among team members. Hence, $e_{A 1}^{*}=e_{A 2}^{*}$ (and $e_{B 1}^{*}=e_{B 2}^{*}$ ) for the cooperative solution.

We can also show that second players choose an effort level which is lower than what a player chooses for an individual production. Moreover, effort choice for individual production is equal to the effort choice in a cooperative social optimum.

Proposition 3 In equilibrium, the optimal level of effort for an individual production is the same as the cooperative socially optimal level of effort for a team production.

Proof See Appendix A. 
This result stems directly from the assumption that the prize $\mathrm{S}$ gives the same individual utility in both cases.

\section{Data and testing strategy}

\subsection{Swimming competitions}

Swimming competitions entail four competitive styles -backstroke, breaststroke, butterfly and freestyle- at varying distances (e.g. 100 meters, 200 meters, etc.) typically in 25 or 50 meter pools. ${ }^{6}$

Relays are a group of swimmers who either all swim freestyle or each swim one different style in the order of backstroke, breaststroke, butterfly and freestyle (medley relay). Except for some specific (usually minor) events, relay teams, according to FINA rules, consist of four swimmers. Unless specified by the Promoter's conditions the nomination of team members and the relay swimming order must be made before the competition. Any relay team member may compete in a race only once. Teams may be changed between heats and finals provided they are from a pool of swimmers whose nomination was submitted for that event. World records are recognized for freestyle relays $4 \times 100$ and $4 \times 200$ meters, and the medley relay $4 \times 100$ meters for either sex in 25 meter and 50 meter courses.

Swimmers in individual competitions and the first swimmer in a relay competition start upon hearing the starting signal. In a relay all the following swimmers start after the previous swimmer touched the wall of the pool. Hence, individual and relay times are comparable only for first swimmers, as there is an advantage for all following swimmers in a relay in terms of reaction time: they can see their teammates coming closer and consequently fine tune their start. In fact, if a first swimmer in a relay competition swims a record time it gets approved.

Various rules guarantee accurate measurement of swimmers' performances. Time keeping is under the supervision of appointed officials and is either made by automatic equipment or manually. If manually registered there are three timekeepers and watches must be certified by the governing body. If two of three watches record the same time, the two identical times are the official time. If all three watches show different times the intermediate time is taken, and if only two of the three watches work the average time is calculated. In any case times are recorded to $1 / 100$ of a second.

\subsection{Data description}

Our dataset was kindly provided by GeoLogix AG, a Suisse company which gets the data directly from the European Swimming Federation (LEN) and other participating federations. In total it comprises 311,784 observations of performances of

\footnotetext{
${ }^{6}$ Rules for these swimming competitions, may they be national or international, are set by the Federation Internationale de Natation (FINA) (www. fina.org).
} 
individual swimmers at more than 7,000 events which took place worldwide between 1972 and 2009. The events included in our sample are both major events such as the Olympic Games, World Championships, European Championships, Pan Pacific Games, the Commonwealth Games or Universiades, and other events, like national championships. Only athletes who took part in the same event and for the same style, both in the individual competition and in the relay, are included.

As for the personal characteristics of the swimmers, we have information on age, gender, nationality, and FINA points. Age is between 6 and 109 years with a median of 16. Gender composition of the sample is more or less equally split. The FINA Point Scoring assigns point values to swimming performances. Points are assigned at every competition, by comparing a swimmer's performance with a base time that is recalculated every year, taking the average of the top ten of the All Time World Rankings. More points go along with better performance. In the sample, FINA points are related to the individual competitions and vary between 5 and 1,181 with a median of 504. Michael Phelps had 1,063 in the year of the Olympic Games in Beijing.

Next we have information on the event (event name, location and beginning and ending day), the competition (style, distance, date of attendance and round - heats, preliminary, semifinals, or finals) with the day of the competition allowing to some extent to control for the sequence of the individual and the relay race, and finally performances, which include the time in the individual and the relay competition, the total relay time, as well as the starting order in the relay and the final placement both for the relay and the individual competition. Table 1 summarizes the data.

\subsection{Empirical strategy}

Our first target for empirical testing is Proposition 1, which suggests that we should be able to detect that exerted effort of players increase towards the end of the competition. In principle, no data on individual competitions are necessary to test this proposition, as it has implications only for the behavior of relay swimmers. However, swimmers are not allocated to a relay in random order: rather, better swimmers are generally placed in the final slot. This implies that later swimmers are on average faster, and attributing this better performance to lower free-riding would be incorrect. To overcome this problem, we use the information on each relay swimmers' performance in the individual competition to control for his/her ability: our dependent variable is therefore the relative difference in swimming time between the relay and the individual competition. If Proposition 1 is of empirical relevance, we should find that this relative difference decreases towards the end of the relay competition. Note that this result is independent of potentially different valuation of prizes between relay and individual competitions, which could explain different effort levels in the two competitions: we say nothing, at this stage, about the sign and magnitude of the relative difference in swimming performances.

However, if we are willing to assume an equal valuation of the price $S$, by Proposition 3 we can interpret the performance in the individual competition as 
the socially optimal level of effort in the relay, and measure the extent of freeriding by comparing individual and relay swimming times. This can be done for first players only, as subsequent players enjoy an advantage in reaction time, as already discussed. By Proposition 2, however, free-riding for subsequent players is reduced but not eliminated.

\section{Empirical evidence}

\subsection{Descriptive evidence}

Table 2 reports the relative difference in swimming time between the relay and the individual competition for different starting orders in the relay. First swimmers are, on average, slower in relays, with respect to their performance in the individual competition. In the whole sample (66,561 observations, with an average swimming time in the individual competition of 56.84 seconds), this difference amounts to $.22 \%$, that is $12 / 100$ of a seconds in absolute terms.

Testing the relative difference for the first swimmers against the null of there being no difference in performance yields a highly significant p-value. This result is robust against splitting the sample along gender or age. It is furthermore valid for swimmers with higher or lower FINA points than the median swimmer. It also holds over all styles if we focus on the sign of the difference in swimming times and in 6 out of 7 subgroups for the various styles in terms of significance.

There are no indications that training or the use of illegal substances targeted to a specific competition (individual or relay) might disturb our results. In $87 \%$ of the observations individual and relay competitions are within 1 day of separation which implies that training efforts influence individual and relay competitions equally. With illegal substances targeting longer term goals such as the building up of red blood cells basically the same logic applies as with legal training methods.

One might also be concerned that fatigue decreases performance in later events. However, the distribution of days of separation between individual and relay competitions is quite symmetric. In any case, we also split the sample along the timing of competitions to check whether it makes a difference if the individual race took place before or after the relay at the particular swimming event for which we compare the swimming times. It is still true that relay performances are weaker than individual performances.

Finally our result is also valid if we look into major events only.

As we have already noted, direct comparison between the relay and the individual competition is possibile only for first swimmers, as subsequent swimmers enjoy an advantage in terms of reaction time, given that they can see the previous swimmer approaching the end of his/her leg. This explains why the time difference turns negative for the second to the forth swimmers in the relay. More importantly, however, last swimmers in relays seem to be faster than swimmers starting 2nd or 3rd, and there seems to be a small difference, in the broad averages, between swimmers starting 3 rd and swimmers starting 2 nd. 
Summarizing these findings, faster swimming times for later swimmers in the relays provide evidence in support of our Proposition 1. Furthermore, if one is willing to accept the hypothesis of equally valuable prizes in the individual and the relay competition, the evidence presented for first swimmers is consistent with the implications of Propositions 2 and 3 of our theoretical model. In order to further elaborate on these findings we turn to a multivariate analysis which allows us to fully exploit our dataset.

\subsection{Regression analysis}

The dependent variable that we use in the multivariate analysis is the relative difference between relay and individual swimming times, as already introduced in table 2. In order to control for the reaction time and to test for our theoretical prediction we introduce dummy variables for the starting order in the relay competition. Other controls are gender, age ${ }^{7}$, style, type of the competition (major vs. non-major) and schedule (whether the individual competition is on a day before the relay, on the same day, or on a day after).

We expect the coefficient of the dummy variable for the first swimmer in the relay to be positive. Furthermore, we expect the coefficients for the dummy variables for subsequent swimmers to be negative because of the advantage in the reaction time for every swimmer following the first one. As later swimmers should on average exert more effort we also expect that the order dummies change in size with higher orders becoming more negative.

This is what we find (as shown in table 3, Model 1): the estimated parameter for the dummy for the first swimmer in the relay is .08 (the constant); second, third and fourth swimmers entail a reduction in the relative time gap of .66, .68 and .95 percentage points, with respect to first swimmers. All dummies are highly significant with p-values smaller than .001 . The Wald tests reject equality of the coefficients of the order two, three and four dummies at a high level of significance. As for the other controls, we find that female swimmers and young and old age groups perform relatively worse in relays, while the gap in relative performance is reduced in major events.

\subsection{Robustness analysis}

We have already dealt with the most important composition issue, the fact that more able swimmers might be placed in a later order slot. The fact that we can control for individual ability -as measured by the performance in the individual competition- solves the problem. However, one might be concerned with the fact that some swimmers might be more motivated than others in swimming a relay,

\footnotetext{
${ }^{7}$ We use three age groups rather than a continuous age variable, in order for the coefficients of the starting order dummy variables to show the effects of the starting order for the reference group (swimmers aged 15-30), rather than for swimmers of a specific age; the consequential reduction in explanatory power -as measured by $\mathrm{R}^{2}$ - is very small.
} 
and that team composition might reflect this. For instance, should team managers put more motivated athletes as the last swimmers in the relay, we would have less motivated first swimmers, something that could explain their slower performance, even after controlling for individual ability. This is a remote possibility, as it is plausible that heterogeneity in ability matters more than heterogeneity in motivation in determining (expected) performance and hence team composition choices. ${ }^{8}$

In order to control for motivational and other (time invariant) unobservable characteristics of our swimmers we introduce individual fixed effects (Model 2). By doing so, we compare the difference one specific athlete scored between relay and individual time when starting the relay at a different order (at different events). For example, a swimmer might have participated at the Olympic Games and the World Championships for $100 \mathrm{~m}$ freestyle and the $4 \times 100$ meters freestyle as third and forth swimmer, respectively. There should be no increase in the relative performance with respect to the individual race when he swam forth, even if results are driven by unobservable characteristics. If, however, we still observe faster swimming times for later swimmers after canceling out unobservable individual characteristics, it would strengthen the finding of free-riding.

Running a fixed effects model yields a coefficient for the first order dummy (the constant) of $.51 .^{9}$ The coefficients for the other order dummies become -.71 , -.78 and -1.03 , respectively. Hence, the fixed effect model confirms our previous findings (and our theoretical model). Note that the number of observations drops to 107,808 as all records pertaining to swimmers for which there is no variation in the independent variables have to be dropped.

One could also object that free-riding depends on the competitive pressure, and that our results are driven by competitions that are either not so close, or of minor importance. Our findings that the relative performances in relays are worse for swimmers at the beginning and at the end of their career, and improve in major events (Model 1) confirm this intuition. As a robustness check, we thus estimate our fixed effect model on finals only, both for the relay and the individual competition (Model 3), on the assumption that finals are more competitive. The coefficient for the first order dummy (the constant) is still positive (.81), while the coefficients for the second, third and fourth order dummy variables are negative, decreasing and significantly different from each other: $-.75,-.83,-1.23$ respectively. Due to the additional restriction, the number of observations drops to 25,138 .

The results still go through if we further restrict to swimmers ending up in the first four positions both in the relay and in the individual competition (the coefficients for the order dummies are, respectively, 1.26, -.94, -1.08, -1.52).

As a final robustness check, in order to reduce a further cause of unobserved heterogeneity, we estimate our fixed effect model on freestyle swimmers only. The

\footnotetext{
${ }^{8}$ Interviews with team managers confirm this intuition.

${ }^{9}$ This value cannot be fully compared with the corresponding value of Model 1, as the age group variable is not included (in a fixed effect model, it would be estimated only on ages around the age group thresholds). Running Model 1 without controlling for age group entails a coefficient for the order_1 dummy (the constant) equal to .18 .
} 
results still go through (the coefficients for the order dummies are, respectively, $.86,-.76,-.85,-1.25)$.

\subsection{Size of the effects}

While the results are supporting our theoretical propositions, one may wonder if these effects are of any meaningful size. In order to get a better understanding of the size of the effects that we detected, we look into the time differences by final placements, i.e. the average lag in swimming time between individuals belonging to teams that finished in $n$th place in relays and individuals belonging to teams that finished in $n-1$ th place, for $n>1$.

This lag is, on average among the first 10 positions in the final ranking, .23\% for major events and $1.35 \%$ for non-major events. These figures can be compared to the ones in table 2. Doing so, we calculated for first swimmers at a major event a relative time difference between relay and individual competition of $0.03 \%$, whereas the relative time difference for all other events amounts to $0.22 \%$.

If we turn to the regression results (table 3, Model 2) we get an impact that is even more relevant: athletes in our reference category are on average .51 percentage points slower in relays than in individual competitions compared to an average lag over an immediately preceding team of .78 percentage points for this group (100m male freestyle, non-major events, swimmer aged 15-30).

Assessing the relevance of the finding that effort decreases as team members get involved in earlier stages of the production process, involves looking at the difference between the coefficients of second, third and fourth order dummies in table 3.

Comparing the estimated effect of the forth swimmers with the third swimmers yields a reduction in free-riding of $1.03-.78=.25$ percentage points. This value must again be compared with an average lag over the preceding team of .78 percentage points, and accounts for one third of this lag. The detected effect between third and second swimmers is smaller $(.78-.71=.07)$, but still sizeable. By comparing the value of the fourth order dummy with that of the second order dummy we get a reduction in free-riding of .32 percentage points, which should be regarded as a lower bound in the overall reduction of free-riding (since it is not possible to assess the reduction between first and second swimmers, due to the presence of a reaction time advantage for first swimmers).

\section{Conclusions}

In this paper we developed a simple model of sequential contributions to a team when teams compete against each other. We show that in such a setting there is free-riding among team members even under competition between teams. We also show that team members contributing earlier to a team's common task contribute less than the team members contributing later. The mechanism underlying the 
result is substitutability of inputs between team members to a Tullock contest. At the margin a team member contributing earlier refrains to increase costly efforts as he can foresee that the following team members will reduce their input so that on aggregate a team's effort for winning the competition would not change. In a cooperative solution and from a social point of view it would be optimal to share the burden equally among team members. But this does not happen even under between-team competition as individuals contribute sequentially to the team.

Drawing on a unique data set of more than 300,000 observation from swimming competitions from all over the world during the last four decades we find evidence for free-riding and the pattern of efforts over the course of sequential contributions to a team as suggested by our model.

The basic idea which we employed was to compare for a given event the swimming performance of individual swimmers for single and relay competitions. By definition no free-riding occurs in a single competition which is why swimmers should exert full effort at these occasions. Taking their performance in the individual race as a control we find that on average these swimmers swim slower in relays. Moreover, controlling for reaction times and individual effects we find that free-riding diminishes as we move from the second, to the third and finally the fourth swimmer in the relays. Results are robust against including various controls. Furthermore, the estimated time differences occur to be of meaningful size.

Our attribution of the lower performances in relay with respect to individual competitions to free-riding depends on the assumption that the prize $S$ is equally valuable in relays and individual competitions. This assumption might be questioned: even with equal monetary prize, obtaining it in an individual competition might be more valuable as the honors do not have to be shared. ${ }^{10}$ If this was the case, it could be socially optimal to individually provide less effort in relays, and our empirical analysis of Propositions 2 and 3 would not allow us to detect any inefficiency. We do not counteract to this remark (although it could be argued that for a vast majority of athletes what matters in winning a gold medal in their career is that they are gold medalist. The difference in utility from winning it in the individual competition rather than in the relay are, if any, of minor importance.) What is more important, however, is that our result that there is free-riding within teams, and that this free-riding is stronger for early contributors, remains unaffected from a potentially different valuation of prizes won in relays and individual competitions.

Given that team production is often unavoidable, and that, as we have shown, neither competition nor an almost ideal monitoring system solve the free-riding problem, our results point to the necessity of devising better incentive mechanisms.

\footnotetext{
${ }^{10}$ The other side of 'two in distress make sorrow less'.
} 


\section{Acknowledgements}

We would like to thank GeoLogix AG for providing us with the data and in particular Christian Kaufmann for his kind and knowledgable support through all stages of this project. We also thank Alois Prinz, Matteo Rizzoli, Alberto Zazzaro and participants of the 37th Annual Conference of the Eastern Economic Association in New York and of the Conference on Tournaments, Contests and Relative Performance Evaluation in Raleigh who commented on earlier versions of the paper. 


\section{Appendix A}

\section{Proposition 1}

Proof The proof is in two parts. In part (I) we show that the f.o.c. define the optimal effort level for players 1 as the s.o.c. are also satisfied. In part (II) we show that the optimal effort level for players 1 is lower than the optimal effort level for players 2.

\section{Part I:}

Expected payoffs for the first player of team $A$ (analogously for team $B$ ) are:

$$
V_{A 1}=p_{A} S-e_{A 1}^{2}
$$

with the f.o.c. as shown in the main text being

$$
\frac{\mathrm{d} V_{A 1}}{\mathrm{~d} e_{A 1}}=\alpha \frac{\left(e_{B 1}+e_{B 2}\right)\left(1+\frac{\mathrm{d} e_{A 2}}{\mathrm{~d} e_{A 1}}-\frac{\mathrm{d} e_{B 2}}{\mathrm{~d} e_{A 1}}\right)}{D^{2}} S-2 e_{A 1}=0 .
$$

The solution to this equation is the optimal choice of effort for player 1, given that the s.o.c. is satisfied. To see that this is indeed the case, consider

$$
\begin{aligned}
\frac{\mathrm{d}^{2} V_{A 1}}{\mathrm{~d} e_{A 1}^{2}} & =\alpha \frac{D^{2}\left(\frac{\mathrm{d} e_{B 2}}{\mathrm{~d} e_{A 1}}\left(1+\frac{\mathrm{d} e_{A 2}}{\mathrm{~d} e_{A 1}}\right)+\left(e_{B 1}+e_{B 2}\right) \frac{\mathrm{d}^{2} e_{A 2}}{\mathrm{~d} e_{A 1}^{2}}\right)}{D^{4}} S \\
& -\alpha \frac{D^{2}\left(\left(1+\frac{\mathrm{d} e_{A 2}}{\mathrm{~d} e_{A 1}}\right) \frac{\mathrm{d} e_{B 2}}{\mathrm{~d} e_{A 1}}+\left(e_{A 1}+e_{A 2}\right) \frac{\mathrm{d}^{2} e_{B 2}}{\mathrm{~d} e_{A 1}^{2}}\right)}{D^{4}} S \\
& -\alpha \frac{\left(\left(e_{B 1}+e_{B 2}\right)\left(1+\frac{\mathrm{d} e_{A 2}}{\mathrm{~d} e_{A 1}}\right)+\left(e_{A 1}+e_{A 2}\right) \frac{\mathrm{d} e_{B 2}}{\mathrm{~d} e_{A 1}}\right) 2 D\left(1+\frac{\mathrm{d} e_{A 2}}{\mathrm{~d} e_{A 1}}+\frac{\mathrm{d} e_{B 2}}{\mathrm{~d} e_{A 1}}\right)}{D^{4}} S-2
\end{aligned}
$$

We can find expressions for $\frac{\mathrm{d}^{2} e_{A 2}}{\mathrm{~d} e_{A 1}^{2}}, \frac{\mathrm{d}^{2} e_{B 2}}{\mathrm{~d} e_{A 1}^{2}}$ and $\frac{\mathrm{d} e_{A 2}}{\mathrm{~d} e_{A 1}}$ by taking the total derivatives around the optimal solution for second players given by the f.o.c.:

$$
\begin{aligned}
& \frac{\partial H}{\partial e_{A 2}} \frac{\mathrm{d} e_{A 2}}{\mathrm{~d} e_{A 1}}+\frac{\partial H}{\partial e_{B 2}} \frac{\mathrm{d} e_{B 2}}{\mathrm{~d} e_{A 1}}+\frac{\partial H}{\partial e_{A 1}}=0 \\
& \frac{\partial F}{\partial e_{A 2}} \frac{\mathrm{d} e_{A 2}}{\mathrm{~d} e_{A 1}}+\frac{\partial F}{\partial e_{B 2}} \frac{\mathrm{d} e_{B 2}}{\mathrm{~d} e_{A 1}}+\frac{\partial F}{\partial e_{A 1}}=0 .
\end{aligned}
$$

Rewriting in matrix form yields

$$
\left(\begin{array}{cc}
\frac{\partial H}{\partial e_{A 2}} & \frac{\partial H}{\partial e_{B} 2} \\
\frac{\partial F}{\partial e_{A 2}} & \frac{\partial F}{\partial e_{B 2}}
\end{array}\right)\left(\begin{array}{c}
\frac{\mathrm{d} e_{A 2}}{\mathrm{~d} e_{A 1}} \\
\frac{\mathrm{d} e_{B 2}}{\mathrm{~d} e_{A 1}}
\end{array}\right)=\left(\begin{array}{c}
-\frac{\partial H}{\partial e_{A 1}} \\
-\frac{\partial F}{\partial e_{A 1}}
\end{array}\right) .
$$


Applying Cramer's rule we get for the effect of a change in the effort of the agent moving first in team $A$ on the optimally chosen effort of the second team member in $A$

$$
\frac{\mathrm{d} e_{A 2}}{\mathrm{~d} e_{A 1}}=\frac{-u v+x w}{y v-z x}
$$

and for the effect on the optimal effort of the second player in team $B$

$$
\frac{\mathrm{d} e_{B 2}}{\mathrm{~d} e_{A 1}}=\frac{-y w+z u}{y v-z x} .
$$

Furthermore we have

$$
\begin{aligned}
\frac{\mathrm{d}^{2} e_{A 2}}{\mathrm{~d} e_{A 1}^{2}} & =\frac{u^{\prime}(u-v)}{v^{2}} \\
\frac{\mathrm{d}^{2} e_{B 2}}{\mathrm{~d} e_{A 1}^{2}} & =\frac{v^{\prime}(v-u)}{v^{2}}
\end{aligned}
$$

with

$$
\begin{gathered}
u \equiv \frac{\partial H}{\partial e_{A 1}}=\alpha \frac{-\left(e_{B 1}+e_{B 2}\right) 2 D}{D^{4}} S<0 \\
v \equiv \frac{\partial F}{\partial e_{B 2}}=\alpha \frac{-\left(e_{A 1}+e_{A 2}\right) 2 D}{D^{4}} S-2<0 \\
w \equiv \frac{\partial F}{\partial e_{A 1}}=\alpha \frac{D^{2}-\left(e_{A 1}+e_{A 2}\right) 2 D}{D^{4}} S=\alpha \frac{1-\frac{2\left(e_{A 1}+e_{A 2}\right)}{D}}{D^{2}} S=0 \\
x \equiv \frac{\partial H}{\partial e_{B 2}}=\alpha \frac{D^{2}-\left(e_{B 1}+e_{B 2}\right) 2 D}{D^{4}} S=\alpha \frac{1-\frac{2\left(e_{B 1}+e_{B 2}\right)}{D}}{D^{2}} S=0 \\
y \equiv \frac{\partial H}{\partial e_{A 2}}=\alpha \frac{-\left(e_{B 1}+e_{B 2}\right) 2 D}{D^{4}} S-2<0 \\
z \equiv \frac{\partial F}{\partial e_{A 2}}=\alpha \frac{D^{2}-\left(e_{A 1}+e_{A 2}\right) 2 D}{D^{4}} S=\alpha \frac{1-\frac{2\left(e_{A 1}+e_{A 2}\right)}{D}}{D^{2}} S=0 \\
u^{\prime} \equiv \frac{\partial^{2} H}{\partial e_{A 1}^{2}}=\alpha \frac{3 S}{D^{3}}>0 \\
v^{\prime} \equiv \frac{\partial^{2} F}{\partial e_{A 1} \partial e_{B 2}}=\alpha \frac{S}{D^{3}}>0 \\
w^{\prime} \equiv \frac{\partial^{2} F}{\partial e_{A 1}^{2}}=-\alpha \frac{S}{D^{3}}<0 \\
x^{\prime} \equiv \frac{\partial^{2} F}{\partial e_{A 1} \partial e_{B 1}}=\alpha \frac{S}{D^{3}}>0 \\
y^{\prime} \equiv \frac{\partial^{2} H}{\partial e_{A 1} \partial e_{A 2}}=\alpha \frac{3 S}{D^{3}}>0 \\
z^{\prime} \equiv \frac{\partial^{2} F}{\partial e_{A 1} \partial e_{A 2}}=-\alpha \frac{S}{D^{3}}
\end{gathered}
$$


Note that $u^{\prime}=y^{\prime}, v^{\prime}=x^{\prime}=-w^{\prime}=-z^{\prime}$. Because of symmetry we have $e_{B 1}+e_{B 2}=e_{A 1}+e_{A 2}$ so that in equilibrium by inserting terms into eq. (18) we get

$$
\frac{\mathrm{d} e_{B 2}}{\mathrm{~d} e_{A 1}}=0
$$

Hence the s.o.c. simplifies to

$$
\frac{\mathrm{d}^{2} V_{A 1}}{\mathrm{~d} e_{A 1}^{2}}=\alpha\left(e_{B 1}+e_{B 2}\right) \frac{D\left(\frac{\mathrm{d}^{2} e_{A 2}}{\mathrm{~d} e_{A 1}^{2}}-\frac{\mathrm{d}^{2} e_{B 2}}{\mathrm{~d} e_{A 1}^{2}}\right)-2\left(1+\frac{\mathrm{d} e_{A 2}}{\mathrm{~d} e_{A 1}}\right)^{2}}{D^{3}} S-2 .
$$

Inserting $\frac{\mathrm{d}^{2} e_{A 2}}{\mathrm{~d} e_{A 1}^{2}}, \frac{\mathrm{d}^{2} e_{B 2}}{\mathrm{~d} e_{A 1}^{2}}$ and $\frac{\mathrm{d} e_{A 2}}{\mathrm{~d} e_{A 1}}$ in equation (35) and further rearranging yields

$$
\frac{\mathrm{d}^{2} V_{A 1}}{\mathrm{~d} e_{A 1}^{2}}=\alpha \frac{2 S}{D^{2}}\left(\alpha \frac{S}{D^{2}}-6\right)-8
$$

which is negative if

$$
\alpha \frac{S}{\left(2 e_{A 1}+2 e_{A 2}\right)^{2}}<6 .
$$

Using equation (8) one can show that the 1.h.s. is never larger than 2 which finally proves that the s.o.c. is fulfilled.

\section{Part II:}

Now, in order to have an interior solution and to show that the second player exerts more effort it must hold that:

$$
-1<\frac{\mathrm{d} e_{A 2}}{\mathrm{~d} e_{A 1}}-\frac{\mathrm{d} e_{B 2}}{\mathrm{~d} e_{A 1}}<0
$$

which follows from the comparison of the f.o.c. of the first and second players. We already know that $\frac{\mathrm{d} e_{B 2}}{\mathrm{~d} e_{A 1}}=0$, see eq. (34), and $\frac{\mathrm{d} e_{A 2}}{\mathrm{~d} e_{A 1}}=-\frac{u}{y}=-\frac{u}{v}$. As $|u|<|v|$ it holds that $-1<\frac{\mathrm{d} e_{A 2}}{\mathrm{~d} e_{A 1}}-\frac{\mathrm{d} e_{B 2}}{\mathrm{~d} e_{A 1}}<0$ which proves our Proposition.

\section{Proposition 2}

Proof Due to symmetry, the choices of competing swimmers of the same order must be identical. From the f.o.c. of second players given in (6) and (7), by substituting $e_{B 1}=e_{A 1}$ and $e_{B 2}=e_{A 2}$, we find that the optimal effort level of second swimmers is implicitly given by:

$$
c^{\prime}\left(e_{A 2}\right)=2 e_{A 2}=\alpha \frac{S}{4\left(e_{A 1}+e_{A 2}\right)}
$$

or, equivalently, by $c^{\prime}\left(e_{B 2}\right)=2 e_{B 2}=\alpha \frac{S}{4\left(e_{B 1}+e_{B 2}\right)}$. 
From a cooperative perspective, given that the prize is non-rival within the team, the optimal level of effort maximizes:

$$
V_{A}=2 p_{A} S-e_{A 1}^{2}-e_{A 2}^{2} .
$$

The f.o.c. with respect to the effort choices $e_{A 1}$ and $e_{A 2}$ for team $A$ (analogously for team $B$ ) are:

$$
\begin{gathered}
\alpha \frac{2\left(e_{B 1}+e_{B 2}\right)}{D^{2}} S-2 e_{A 2}=0 \\
\alpha \frac{2\left(e_{B 1}+e_{B 2}\right)}{D^{2}} S-2 e_{A 1}=0 .
\end{gathered}
$$

Symmetry then implies

$$
2 e_{A 1}=2 e_{A 2}=\alpha \frac{S}{2\left(e_{A 1}+e_{A 2}\right)}=\alpha \frac{S}{4 e_{A 2}}
$$

which is higher than the effort exerted by the second player in the non-cooperative solution given by (39).

\section{Proposition 3}

Proof From equation (39), absent player 1, player 2's optimal effort would be determined by:

$$
c^{\prime}\left(e_{A 2}\right)=2 e_{A 2}=\alpha \frac{S}{4 e_{A 2}}
$$

which is the same as the socially optimal effort in team production. 


\section{References}

A.A. Alchian and H. Demsetz. Production, information costs, and economic organization. American Economic Review, 62:777-795, 1972.

J. Andreoni, P.M. Brown, and L. Vesterlund. What makes an allocation fair? some experimental evidence. Games and Economic Behavior, 40:1-24, 2002.

G. Bornstein, I. Erev, and O. Rosen. Intergroup competition as a structural solution for social dilemma. Social Behavior, 5:247-260, 1990.

L. C. Corchón. The theory of contests: a survey. Review of Economic Design, 11: 69-100, 2007.

R. Ehrenberg and M. Bognanno. Do tournaments have incentive effects? Journal of Political Economy, 98:1307-1324, 1990.

I. Erev and A. Rapoport. Provision of step-level public goods: The sequential contribution mechanism. The Journal of Conflict Resolution, 34:401-425, 1990.

I. Erev, G. Bornstein, and R. Galili. Constructive intergroup competition as a solution to the free rider problem: a field experiment. Journal of Experimental Social Psychology, 29:463-478, 1993.

J.J. Everett, R.E. Smith, and K.D. Williams. Effects of team cohesion and identifiability on social loafing in relay swimming performance. International Journal of Sport Psychology, 23:311-324, 1992.

Simon Gaechter, Daniele Nosenzo, Elke Renner, and Martin Sefton. Sequential versus simultaneous contributions to public goods: Experimental evidence. CESifo Working Paper Series 2602, CESifo, 2009.

M. Gradstein. Rent seeking and the provision of public goods. Economic Journal, 103:1236-1243, 1993.

A. Gunnthorsdottir and A. Rapoport. Embedding social dilemmas in intergroup competition reduces free-riding. Organizational Behavior and Human Decision Processes, 101:184-199, 2006.

B. Holmstrom. Moral harzard in teams. The Bell Journal of Economics, 13:324340, 1982.

H. Jia. A stocastic derivation of the ratio form of contest success functions. Public Choice, 135:125-130, 2008.

L. M. Kahn. The sports business as a labor market laboratory. Journal of Economic Perspectives, 14(3):75-94, 2000. 
E. Katz, S. Nitzan, and J. Rosenberg. Rent-seeking for pure public goods. Public Choice, 65:49-60, 1990.

K. Konrad. Strategy and Dynamics in Contests. Oxford University Press, New York, 2009.

E. E. Lawler, S. A. Mohrman, and G. Benson. Organizing For High Performance: Employee Involvement, TQM, Reengineering, And Knowledge Management in the Fortune 1000. Jossey-Bass, San Francisco, 2001.

E. P. Lazear and K. L. Shaw. Personnel economics: the economist's view of human resources. Journal of Economic Perspectives, 21:91-114, 2007.

J.A. Miles and J. Greenberg. Using punishment threats to attenuate social loafing effects among swimmers. Organizational Behavior and Human Decision Processes, 56:246-265, 1993.

S. Skaperdas. Contest success functions. Economic Theory, 7:283-290, 1996.

R.M. Sorrentino and B.H. Sheppard. Effects of affiliation-related motives on swimmers in individual versus group competition: a field experiment. Journal of Personality and Social Psychology, 36:704-714, 1978.

S. Szymanski. The assessment: the economics of sport. Oxford Review of Economic Policy, 19:467-477, 2003.

G. Tullock. Efficient rent-seeking. In J. M. Buchanan, R.D. Tollison, and G. Tullock, editors, Towards a theory of a rent-seeking society, page pp97. Texas A\&M University Press, College Station, 1980.

H. W. Ursprung. Public goods, rent dissipation, and candidate competition. Economics and Politics, 2:115-132, 1990.

Har R. Varian. Sequential contributions to public goods. Journal of Public Economics, 53:165-186, 1994.

K.D. Williams, S.A Nida, L.D. Baca, and B. Latané. Social loafing and swimming: effects of identifiability on individual and relay performance of intercollegiate swimmers. Basic and Applied Social Psychology, 10:73-81, 1989. 
Table 1: Descriptive statistics

\begin{tabular}{|c|c|c|c|}
\hline \multicolumn{4}{|l|}{ Variable } \\
\hline Number of events & & 7,081 & \\
\hline Overall no. of observations & & 311,784 & \\
\hline Olympic games & & 660 & \\
\hline World championships & & 1,531 & \\
\hline European championships & & 766 & \\
\hline Pan Pacific games & & 390 & \\
\hline Commonwealth games & & 184 & \\
\hline Universiades & & 304 & \\
\hline \multicolumn{4}{|l|}{ Style } \\
\hline $50 \mathrm{~m}$ Breaststroke & & 20,092 & \\
\hline 50m Fly & & 17,619 & \\
\hline 50m Freestyle & & 103,086 & \\
\hline $100 \mathrm{~m}$ Breaststroke & & 28,719 & \\
\hline 100m Fly & & 25,184 & \\
\hline 100m Freestyle & & 96,034 & \\
\hline 200m Freestyle & & 21,050 & \\
\hline \multicolumn{4}{|l|}{ Schedule } \\
\hline individual first $^{(a)}$ & & 80,825 & \\
\hline relay first ${ }^{(b)}$ & & 83,292 & \\
\hline same day & & 147,667 & \\
\hline \multicolumn{4}{|l|}{ Round (individual competition) } \\
\hline timed finals (default) & & 144,436 & \\
\hline finals & & 75,862 & \\
\hline semi-finals & & 2,630 & \\
\hline preliminars & & 88,596 & \\
\hline others $^{(c)}$ & & 260 & \\
\hline \multicolumn{4}{|l|}{ Round (relay) } \\
\hline timed finals (default) & & 202,384 & \\
\hline finals & & 94,807 & \\
\hline preliminars & & 14,025 & \\
\hline others $^{(c)}$ & & 568 & \\
\hline \multicolumn{4}{|l|}{ Order (relay) } \\
\hline $1 \mathrm{st}$ & & 66,551 & \\
\hline $2 \mathrm{nd}$ & & 88,593 & \\
\hline $3 \mathrm{rd}$ & & 80,004 & \\
\hline 4 th & & 76,636 & \\
\hline \multicolumn{4}{|l|}{ Gender } \\
\hline male & & 155,341 & \\
\hline female & & 156,443 & \\
\hline & Median & Min & Max \\
\hline Age & 16 & 6 & 109 \\
\hline FINA points & 504 & 5 & 1,181 \\
\hline
\end{tabular}

(a) Day of individual competition before day of relay

(b) Day of individual competition after day of relay

(c) Swim-Off after semi-finals, Swim-Off after preliminars 
Table 2: Comparing individual and relay swimming times only

\begin{tabular}{|c|c|c|c|c|c|c|}
\hline & \multicolumn{6}{|c|}{ Swimming times } \\
\hline & \multirow{2}{*}{$\begin{array}{c}\text { Individual } \\
\text { (secs.) } \\
\text { I }\end{array}$} & \multicolumn{5}{|c|}{$\begin{array}{c}\text { Relative difference } \mathrm{b} / \mathrm{w} \text { relay and individual } \\
(\%)\end{array}$} \\
\hline & & \multicolumn{2}{|c|}{$\frac{R_{1}-I}{I}$} & $R_{3}-I$ & $\frac{R_{3}-I}{I}$ & \multirow{2}{*}{$\frac{\frac{R_{4}-I}{I}}{-.75}$} \\
\hline Overall & 56.84 & .22 & $* * *$ & -.33 & -.34 & \\
\hline Gender & & & & & & \\
\hline male & 53.66 & .20 & $* * *$ & -.43 & -.42 & -.82 \\
\hline female & 58.01 & .23 & $* * *$ & -.25 & -.26 & -.69 \\
\hline Age (yrs) & & & & & & \\
\hline$<15$ & 54.91 & .36 & $* * *$ & -.03 & -.02 & -.47 \\
\hline $15-30$ & 58.47 & .11 & $* * *$ & -.48 & -.51 & -.92 \\
\hline$>30$ & 38.78 & .30 & $* * *$ & -.58 & -.51 & -.95 \\
\hline Ability of swimmer ${ }^{(a)}$ & & & & & & \\
\hline$\leq$ median & 52.64 & .27 & $* * *$ & -.19 & -.17 & -.59 \\
\hline$>$ median & 59.03 & .17 & $* * *$ & -.48 & -.53 & -.92 \\
\hline Style & & & & & & \\
\hline $50 \mathrm{~m}$ Breaststroke & 39.67 & .44 & $* * *$ & -.51 & -.12 & -.25 \\
\hline 50m Fly & 33.58 & .62 & $* * *$ & -.10 & -.48 & -.32 \\
\hline 50m Freestyle & 32.03 & .32 & $* * *$ & -.70 & -.70 & -1.14 \\
\hline $100 \mathrm{~m}$ Breaststroke & 78.27 & .32 & $* * *$ & -.05 & -.05 & -.22 \\
\hline 100m Fly & 67.88 & .05 & & -.53 & -.04 & -.47 \\
\hline 100m Freestyle & 63.08 & .14 & $* * *$ & -.30 & -.31 & -.45 \\
\hline 200m Freestyle & 128.53 & .07 & $* * *$ & -.03 & -.08 & -.04 \\
\hline Event importance & & & & & & \\
\hline major events ${ }^{(b)}$ & 70.99 & .03 & & -.53 & -.56 & -.90 \\
\hline others & 55.66 & .22 & $* * *$ & -.33 & -.34 & -.75 \\
\hline Schedule of competitions & & & & & & \\
\hline individual first $^{(c)}$ & 54.81 & .19 & $* * *$ & -.39 & -.41 & -.98 \\
\hline relay first ${ }^{(d)}$ & 59.81 & .15 & $* * *$ & -.49 & -.49 & -.77 \\
\hline same day & 54.17 & .27 & $* * *$ & -.22 & -.21 & -.63 \\
\hline
\end{tabular}

$I$ - individual competition swimming time

$R_{1}, \cdots, R_{4}$ - relay swimming time, starting order $1, \cdots, 4$

(a) As measured by FINA points

(b) Olympic, Pan Pacific and Commonwealth Games, World and European Championships, Universiades

(c) Day of individual competition before day of relay

(d) Day of individual competition after day of relay

$* * * p<.01$ 
Table 3: Regression results

\begin{tabular}{|c|c|c|c|c|c|c|}
\hline Dependent variable & Model 1 & & $\begin{array}{l}\text { Model } 2 \\
\quad(R-I)\end{array}$ & & Model 3 & \\
\hline constant & .08 & $* * *$ & .51 & $* * *$ & .81 & $* * *$ \\
\hline order_2 & -.66 & $* * *$ & -.71 & $* * *$ & -.75 & $* * *$ \\
\hline order_3 & -.68 & $* * *$ & -.78 & $* * *$ & -.83 & $* * *$ \\
\hline order_4 & -.95 & $* * *$ & -1.03 & $* * *$ & -1.23 & $* * *$ \\
\hline female & .07 & $* * *$ & & & & \\
\hline age $<15$ & .52 & $* * *$ & & & & \\
\hline age $>30$ & .26 & $* * *$ & & & & \\
\hline major & -.19 & $* * *$ & -.14 & $* * *$ & -.31 & $* * *$ \\
\hline same day & .19 & $* * *$ & .10 & $* * *$ & .02 & \\
\hline relay first & -.02 & $* *$ & -.04 & $* * *$ & .02 & \\
\hline 50m Breastroke (5) & -.27 & $* * *$ & -.46 & $* * *$ & -.61 & $* * *$ \\
\hline 50m Fly (6) & -.22 & $* * *$ & -.37 & $* * *$ & -.42 & $* * *$ \\
\hline 50m Freestyle (7) & -.48 & $* * *$ & -.72 & $* * *$ & -.61 & $* * *$ \\
\hline 100m Breastroke (1) & .27 & $* * *$ & .32 & $* * *$ & .27 & $* * *$ \\
\hline 100m Fly (2) & .33 & $* * *$ & .39 & $* * *$ & .41 & $* * *$ \\
\hline 200m Freestyle (4) & .21 & $* * *$ & .31 & $* * *$ & .25 & $* * *$ \\
\hline Fixed effects & No & & Yes & & Yes & \\
\hline R-squared & 0.09 & & .50 & & .56 & \\
\hline \multicolumn{7}{|l|}{ Wald test (F value) } \\
\hline order_2=order_3 & 3.93 & $* *$ & 30.88 & $* * *$ & 5.67 & $* *$ \\
\hline order_3 $=$ order_4 & 822.12 & $* * *$ & 522.59 & $* * *$ & 155.93 & $* * *$ \\
\hline Obs. & 311,784 & & 107,808 & & 25,138 & \\
\hline Notes & & & & & finals $\mathrm{c}$ & \\
\hline
\end{tabular}

Reference category: $100 \mathrm{~m}$ Freestyle, age group 15-30, individual competition on a day prior to the relay, first swimmers 